\title{
Cervical chylous leakage following esophagectomy that was successfully treated by intranodal lipiodol lymphangiography: a case report
}

\author{
Tatsuro Tamura ${ }^{1 *}$, Naoshi Kubo², Akira Yamamoto ${ }^{3}$, Katsunobu Sakurai ${ }^{2}$, Takahiro Toyokawa ${ }^{1}$, Hiroaki Tanaka ${ }^{1}$,
} Kazuya Muguruma', Masakazu Yashiro' ${ }^{1}$ Kiyoshi Maeda', Kosei Hirakawa' and Masaichi Ohira'

\begin{abstract}
Background: Chylous leakage is a well-known complication after esophagectomy, but cervical chylous leakage is relatively rare, and considerable controversy remains regarding the appropriate management strategies. We herein report a case of cervical chylous leakage treated successfully by lipiodol lymphangiography.

Case presentation: The patient, a 70-year-old man with middle thoracic esophageal cancer, underwent radical esophagectomy with 3-field lymph node dissection and subsequently developed cervical chylous leakage. From the second postoperative day (POD2), the amount of fluid in the cervical drainage tube increased by $200-300 \mathrm{ml} /$ day. We started octreotide (300 $\mathrm{\mu g} /$ day) on POD5 and etilefrine $(120 \mathrm{mg} /$ day) on the POD6. However, the amount of cervical discharge did not decrease. We performed lipiodol lymphangiography on POD8. Thereafter, the amount of cervical discharge finally began to decrease. We removed the drainage tube on POD13, and the patient was discharged from the hospital on POD23.
\end{abstract}

Conclusions: Our case suggests the clinical efficacy of lipiodol lymphangiography for cervical chylous leakage after esophagectomy.

Keywords: Esophageal cancer, Cervical chylous leakage, Lipiodol lymphangiography

\section{Background}

Chylous leakage is a complication that may occur after esophagectomy, as a result of intraoperative injury or inadequate ligation of the thoracic duct, thus leading to chylous leakage. The reported incidence of this complication varies between 1.3 and $3.4 \%$ after procedures involving the thorax and neck $[1,2]$. Undiagnosed postoperative chylous leakage may lead to malnutrition, sepsis, and a high mortality rate $[3,4]$. Therefore, a prompt diagnosis and optimum management are required. Recently, etilefrine and octreotide have been reported to be effective in treating chylous leakage. However, conclusive findings regarding the efficacy of this regimen have yet to be obtained.

\footnotetext{
*Correspondence: tamura.tatsuro@med.osaka-cu.ac.jp 'Department of Surgical Oncology, Osaka City University, Graduate School of Medicine, 1-4-3 Asahi-machi, Abeno-ku, Osaka, Japan

Full list of author information is available at the end of the article
}

We herein report a case of esophagectomy complicated by cervical chylous leakage that we treated successfully with lipiodol lymphangiography.

\section{Case presentation}

A 70-year-old man was referred to our hospital for the surgical management of a tumor in the thoracic upper esophagus. A physical examination and laboratory data revealed no remarkable findings. Computed tomography (CT) revealed that the tumor, which was located in the upper thoracic esophagus, had not locally invaded other organs, but there was a metastasis in the right recurrent laryngeal nerve lymph node. Upper gastrointestinal endoscopy showed a 2.5 -cm-long ulcerated type 2 tumor in the upper thoracic esophagus. The extent of tumor invasion was estimated to be T2. A histopathological examination of a biopsy specimen revealed the lesion to 
be squamous cell carcinoma. Pathologic findings showed squamous cell carcinoma of the esophagus, (cT2, N1, M0, cStage II), determined based on the Japanese Classification of Esophageal Cancer, 10th edition. Neoadjuvant chemotherapy consisting of cisplatin and 5-fluorouracil (FP) was performed before surgery. The patient received 2 cycles of the following regimen: intravenous infusions of cisplatin $\left(80 \mathrm{mg} / \mathrm{m}^{2}\right)$ on Day 1 and continuous intravenous infusion of 5-fluorouracil $\left(800 \mathrm{mg} / \mathrm{m}^{2}\right)$ from Days 1 to 5 , every 4 weeks. The tumor and lymph node metastasis were reduced after two cycles, and the neoadjuvant chemotherapy evaluation was deemed a partial response (PR).

The patient underwent subtotal esophagectomy and three-field lymphadenectomy (mediastinal lympadenectomy accompanied with neck and abdominal lymphadenectomy) via thoracoscopy and hand-assisted laparoscopy, and esophagogastrostomy was performed for reconstruction at the neck via the retrosternal route. The thoracic duct was preserved, because the thoracic duct did not approach the tumor and there was no remarkable damage to the thoracic duct during the operation. The histological diagnosis was moderately differentiated squamous cell carcinoma of the esophagus (pT1b, N1, M0, pStage II) based on the Japanese Classification of Esophageal Cancer, 10th edition.

Postoperatively (Fig. 1), a large amount of cervical effusion discharge (100-450 ml/day) flowed continuously from the cervical drainage tube. From the second postoperative day (POD2), due to the amount of discharge from cervical drainage tube increasing by $200-300 \mathrm{ml} /$ day, we suspected chylous leakage at the neck point and administered an elemental diet (Elental) via an intestinal fistula catheter. But the amount of discharge did not decrease. We started octreotide ( $300 \mu \mathrm{g} /$ day) on the POD5 and etilefrine $(120 \mathrm{mg} /$ day) on POD6. The amount of discharge decreased only slightly, so we consulted a radiologist at our hospital and performed lipiodol lymphangiography on POD8. The bilateral inguinal lymph nodes were directly accessed under ultrasound guidance with a 23-gauge needle. Lipiodol was manually injected at about $1 \mathrm{ml}$ per $5 \mathrm{~min}$. The lipiodol injection was observed under fluoroscopic guidance in order to confirm appropriate access by the needle (Fig. 2a). After approximately $10 \mathrm{ml}$ of lipiodol had been injected, a fluoroscopic image showed accumulation of the lipiodol at the ductus thoracicus (Fig. 2b).

A day after lymphangiography, CT showed the accumulation of lipiodol at the left venous angle (Fig. 3). After lymphangiography, the amount of discharge from the cervical drainage tube decreased immediately. On POD9 (1 day after lymphangiography), the patient resumed normal enteral nutrition via the intestinal fistula catheter. On POD10 (2 days after lymphangiography), the cervical discharge disappeared. On POD13 (5 days after lymphangiography), the drainage tube was removed. Cervical discharge was not seen at all after the removal of the tube. On POD22 (14 days after lymphangiography), the patient was discharged home.

\section{Discussion}

Postoperative chylous leakage is a rare but well-known complication of general surgery. The reported incidence of this complication varies between 1.3 and $3.4 \%$ after procedures involving the thorax and neck $[1,2]$. Prophylactic ligation of thoracic duct during the radical resection of esophageal cancer is usually used to prevent and treat chylous leakage. But, there is dispute about prophylactic ligation of the thoracic duct. Fu et al. showed a statistically significant increase in postoperative chylous leakage following ligation, suggesting that prophylactic thoracic ligation was not only unnecessary but also harmful [3].

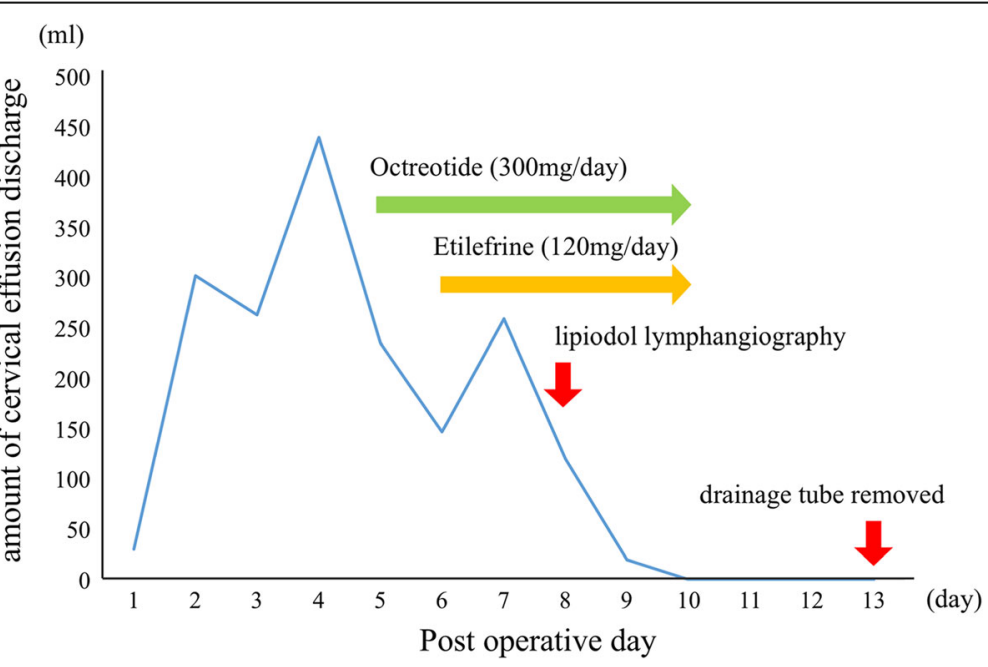

Fig. 1 Postoperative progress and amount of discharge from cervical drainage tube 


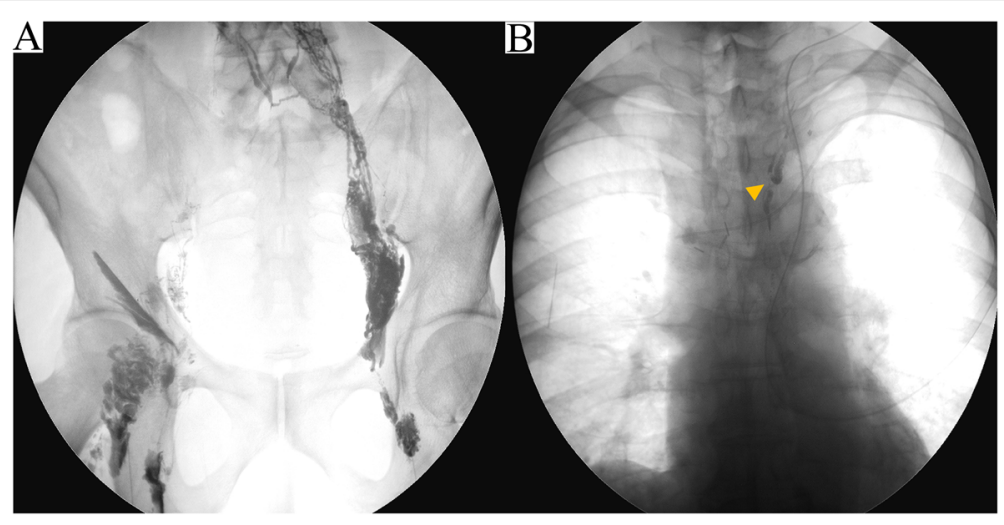

Fig. 2 Lipiodol lymphangiography. The injected lipiodol flowed up through the lymph duct via the bilateral inguinal lymph nodes (a). A fluoroscopic image obtained after $10 \mathrm{ml}$ of lipiodol was injected shows the accumulation of lipiodol at the ductus thoracicus (arrowhead) (b)

Basically, we preserve the thoracic duct other than the case that a tumor approaches it.

Chylous leakage can be life-threatening because of the significant loss of fluid, plasma protein, fat, and immunoregulatory lymphocytes, and affected patients exhibit clinical features of severe malnutrition, hyponatremia, acidosis, hypocalcaemia and susceptibility to infection. Therefore, the mortality rate is high in patients with uncontrolled chylous leakage $[4,5]$. The initial treatment of chylous leakage is usually conservative therapy, such as fasting, a modified diet (low-fat, medium-chain triglycerides; parenteral nutrition; and supplemental elements), drainage of effusion, and the administration of octreotide $[6,7]$ and etilefrine [8]. If conservative therapy fails, surgical interventions such as thoracic duct ligation should be considered. However, the thoracic duct and site of chylous leakage are often difficult to locate during reoperation. In addition, because such surgery is considered to increase the risk of complications, great care must be taken during reoperation.

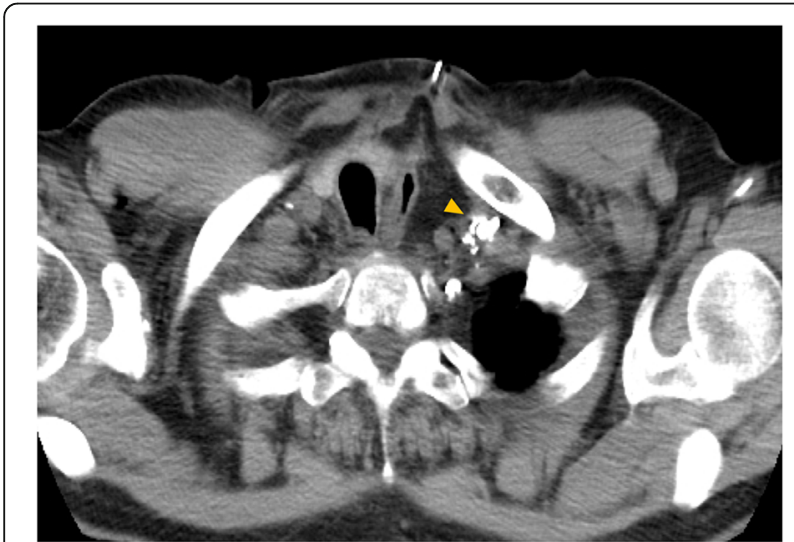

Fig. 3 Coronal sections by CT obtained a day after lipiodol lymphangiography show the accumulation of lipiodol at the left venous angle (arrowhead)
Lipiodol lymphangiography is traditionally a diagnostic tool for identifying chylous leakage, allowing for the detection of the leakage point if surgical intervention is needed [5, 9]. However, lipiodol lymphangiography can also be used as a treatment method itself and not merely to obtain a diagnosis. Even when pleural drainage exceeded $500 \mathrm{ml} /$ day, lipiodol lymphangiography proved an effective treatment in $35 \%$ of patients with chylothorax. The successful treatment rate in patients with failed non-surgical treatment was reported to be $51 \%$ [10]. Matsumoto et al. reported that $89 \%$ patients with postoperative chylous leakage needed no surgical reintervention and that chylous leakage stopped after lipiodol lymphangiography [5]. The mechanism of chylous leakage cessation after lipiodol lymphangiography was suspected to involve the accumulation of lipiodol at the leakage point, inducing an inflammatory reaction and acting as an embolic agent [10].

The traditional procedure of lymphangiography involves the subcutaneous injection of an oily contrast medium, such as lipiodol, into each foot. The traditional procedure has some problems. First by, it is an invasive procedure requiring dorsal incisions, so that it has the potential to lead infection associated with the incisions. Secondly, it is technically challenging because it requires the isolation and cannulation of the fine pedal lymphatic vessels. Even after successful placement of lymphangiogram needles, minimal movement of the patient can dislodge the needles. Whereas, Intranodal lymphangiography using ultrasound is a less-invasive approach in terms of requiring no incision compared to the traditional procedure. Intranodal lymphangiography is simpler and easier because an inguinal node is directly accessed under ultrasound guidance with a 23- gauge to 26-gauge spinal needle with use of a local anesthesia $[11,12]$.

We performed lipiodol lymphangiography using ultrasound-guided bilateral inguinal lymph node puncture in our patient with cervical chylous leakage 
following esophagectomy and successfully treated his cervical chylous leakage. Although there are several reports describing lipiodol lymphangiography as effective against thoracic chylous leakage, few have described the effective treatment of cervical chylous leakage using this technique. In the otolaryngology region, Neel et al. reported a case that was treated against the cervical chylous leakage after laryngectomy with bilateral selective neck dissection using bilateral pedal lymphangiography with iodinated oily contrast medium [13]. But there are few other reports that were treated with lymphangiography, there are many reports treated with thoracic duct ligation for cervical chylous leakage in after the head and neck surgery. Our case demonstrated that lipiodol lymphangiography using ultrasoundguided bilateral inguinal lymph node puncture can indeed be an effective conservative treatment not only for thoracic chylous leakage, but also for cervical chylous leakage.

The limitations of a single case report must be acknowledged; although the proposed benefits of lipiodol lymphangiography by bilateral inguinal lymph node puncture may indeed increase the utilization of thoracic duct embolization, firm conclusions regarding its safety and efficacy require further investigation in a prospective series.

Cervical chylous leakage is relatively rare, and considerable controversy remains regarding the appropriate management strategies. Our case suggests the clinical efficacy of lipiodol lymphangiography for cervical chylous leakage after esophagectomy.

\section{Acknowledgements}

Not applicable.

Funding

No funding.

\section{Availability of data and materials}

The datasets supporting the conclusions of this article are included within the article.

\begin{abstract}
Authors' contributions
All authors participated in the management of the patient in this case report. TT perfomed literature review and drafted the manuscript. NK supervised the case and also supervised the writing of the manuscript. $\mathrm{MO}$ is a chairperson of our department and supervised the entire process. All authors read and approved the manuscript.
\end{abstract}

\section{Competing interests}

The authors declare that they have no competing interests.

\section{Consent for publication}

Written informed consent was obtained from the patient for the publication of this case report and any accompanying images. A copy of the written consent is available for review by the Editor of this journal.

Ethics approval and consent to participate

Not applicable.

\section{Author details}

'Department of Surgical Oncology, Osaka City University, Graduate School of Medicine, 1-4-3 Asahi-machi, Abeno-ku, Osaka, Japan. ${ }^{2}$ Departments of Gastroenterological Surgery, Osaka City General Hospital, 2-13-22 Miyakojima Hondori Miyakojimaku, Osaka 534-0021, Japan. ${ }^{3}$ Department of Diagnostic and Interventional Radiology, Osaka City University, Graduate School of Medicine, 1-4-3 Asahi-machi, Abeno-ku, Osaka, Japan.

Received: 24 December 2016 Accepted: 22 February 2017

Published online: 28 February 2017

\section{References}

1. Kaburagi T, Takeuchi H, Oyama T, Nakamura R, Takahashi T, Wada N, Saikawa Y, Kamiya S, Tanaka M, Wada T, Kitagawa Y. Intraoperative fluorescence lymphography using indocyanine green in a patient with chylothorax after esophagectomy: report of a case. Surg Today. 2013;43(2):206-10.

2. Paul S, Altorki NK, Port JL, Stiles BM, Lee PC. Surgical management of chylothorax. Thorac Cardiovasc Surg. 2009;57(4):226-8.

3. Fu JH, Hu Y, Huang WZ, Yang H, Zhu ZH, Zheng B. [Evaluating prophylactic ligation of thoracic duct during radical resection of esophageal carcinoma]. Ai Zheng. 2006;25(6):728-30.

4. Parvinian A, Mohan GC, Gaba RC, Saldanha DF, Knuttinen MG, Bui JT, Minocha J. Ultrasound-guided intranodal lymphangiography followed by thoracic duct embolization for treatment of postoperative bilateral chylothorax. Head Neck. 2014;36(2):E21-4.

5. Matsumoto T, Yamagami T, Kato T, Hirota T, Yoshimatsu R, Masunami T, Nishimura T. The effectiveness of lumphangiography as a treatment method for various chyle leakages. Br J Radiol. 2009;82(976):286-90.

6. Epaud R, Dubern B, Larroquet M, Tamalet A, Guillemot N, Maurage C, Clement A, Fauroux B. Thetapeutic strategies for idiopathic chylothorax. J Pediatr Surg. 2008:43(3):461-5.

7. Snow AL, Uller W, Kim HB, Alomari Al. Percutaneous embolization of a chylous leak from thoracic duct injury in a child. Cardiovasc Intervent Radiol. 2014;37(4):1111-3.

8. Ohkura Y, Ueno M, lizuka T, Haruta S, Tanaka T, Udagawa H. New combined medical treatment with etilefrine and octreotide for chylothorax after esophagectomy. Medicine. 2015;94(49):e2214.

9. Nagan $\mathrm{H}$, Fok M, Wong J. The role of lymphography in chylothorax following thoracic surgery. Br J Radiol. 1988;61(731):1032-6.

10. Alejandre-Lafont E, Krompiec C, Rau WS, Krombach GA. Effectiveness of therapeutiv lymphography on lymphatic leakage. Acta Radiol. 2011;52(3):305-11.

11. Nadolski GJ, Itkin M. Feasibility of ultrasound-guided intranodal lymphangiogram for thoracic duct embolization. J Vasc Interv Radiol. 2012;23(5):613-6.

12. Yamamoto M, Miyata H, Yamasaki M, Maeda N, Miyazaki Y, Takahashi T, Kurokawa Y, Nakajima K, Takiguchi S, Mori M, Doki Y. Chylothorax after esophagectomy cured by intranodal lymphangiography: a case report. Anticancer Res. 2015:35(2):891-5.

13. Patel N, Lewandowski RJ, Bove M, Nemcek Jr AA, Salem R. Thoracic ductembolization: a new treatment for massive leak after neck dissection. Laryngoscope. 2008;118(4):680-3.

Submit your next manuscript to BioMed Central and we will help you at every step:

- We accept pre-submission inquiries

- Our selector tool helps you to find the most relevant journal

- We provide round the clock customer support

- Convenient online submission

- Thorough peer review

- Inclusion in PubMed and all major indexing services

- Maximum visibility for your research

Submit your manuscript at www.biomedcentral.com/submit
Biomed Central 